\title{
MicroRNA-93 may control vascular endothelial growth factor $A$ in circulating peripheral blood mononuclear cells in acute Kawasaki disease
}

\author{
Kazuyoshi Saito', Hideyuki Nakaoka', Ichiro Takasaki², Keiichi Hirono', Seiji Yamamoto³, Koshi Kinoshita4, Nariaki Miyao', \\ Keijiro Ibuki', Sayaka Ozawa', Kazuhiro Watanabe', Neil E. Bowles ${ }^{5}$ and Fukiko Ichida'
}

\begin{abstract}
BACKGROUND: Kawasaki disease (KD) is the most common systemic vasculitis syndrome primarily affecting medium-sized arteries, particularly the coronary arteries. Though KD may be associated with immunological problems, the involvement of microRNAs (miRs) has not been fully described.

METHODS: We enrolled $23 \mathrm{KD}$ patients and 12 controls. We performed miR and mRNA microarray analysis of peripheral blood mononuclear cells (PBMCs) isolated from acute KD patients and controls. Continuously, we measured specific miRs, mRNA and the expression of proteins by using reversetranscriptase PCR (RT-PCR) and enzyme-linked immunosorbent assay (ELISA).
\end{abstract}

RESULTS: We identified strikingly high levels of miR-182 and miR-296-5p during the acute febrile phase, and of miR-93, miR145-5p, miR-145-3p, and miR-150-3p in the defervescence stage, especially in refractory KD patients. The expression of vascular endothelial growth factor A (VEGFA) mRNA, previously reported to be controlled by miR-93, was significantly elevated during the febrile phase and normalized upon treatment, negatively correlating with the expression of miR-93. Further, plasma levels of VEGF-A correlated with PBMC VEGFA mRNA expression.

CONCLUSION: Several miRs are highly specific to the acute phase of KD, and may participate in regulating the expression of genes in pathways associated with KD. In particular, miR-93 may participate in regulating expression of VEGF-A and contribute to the pathogenesis of arteritis in acute KD.

K awasaki disease (KD) is the most common systemic vasculitis syndrome primarily affecting medium-sized arteries, particularly the coronary arteries (1). KD may be associated with dysregulation of innate immunity and peripheral blood mononuclear cells (PBMCs)-derived vascular endothelial growth factor A (VEGF-A) may contribute to later vascular injury and remodeling in $\mathrm{KD}(2,3)$. Although the etiology of $\mathrm{KD}$ is not yet clear, current theories center on innate immunological problems
(4-8), and several signaling pathways, including transforming growth factor- $\beta$ (TGF- $\beta$ ), Nuclear factor of kappa B (NF- $\kappa B$ ), and VEGF, have been reported to be involved in immune dysregulation and the pathogenesis of acute $\mathrm{KD}(2,9)$. VEGF-A is well known to have a role in angiogenesis, in vasodilation by indirect nitric oxide release, and in the chemotactic function of macrophages and granulocytes $(10,11)$. We previously reported that serum levels of VEGF-A correlate with the development of coronary arterial lesions (CAL) in KD patients (3).

MicroRNA (miRs) are small noncoding RNAs, 18-25 nucleotides in length, that mediate gene silencing through imperfect hybridization to 3' untranslated regions in target mRNAs and modulate a variety of biological activities including immunological reactions. MiRs influence gene expression post-transcriptionally via translational repression and/or mRNA degradation. Although the prevalence and sway of miR-mediated regulation in diverse cellular processes has become increasingly apparent, elucidation of miR regulatory mechanisms is often challenging due in part to miRs commonly exhibiting slight effects on an array of targets. The human genome encodes more than 1000 different miRs that are expressed in a highly dynamic manner in many organs (12-14). For example, miR-146 and miR-150 regulate NF- $\kappa B-$ related inflammation (15). Arteritis in $\mathrm{KD}$ is known to be involved NF- $\mathrm{kB}$ signaling $(9,16)$ but the involvement of miRs in KD has not been fully described.

We hypothesized that miRs have a role in regulating PBMCrelated immune responses during the acute phase of KD and analyzed the expression of miRs and mRNAs during this phase of KD to determine if we could identify miRs that modulate gene expression involved in key signaling pathways and could be related to pathogenesis.

\section{RESULTS \\ Patient Characteristics and Demographics}

The demographic data of the KD patients, the febrile group and the healthy controls are shown in Table 1 . There were no significant differences between the KD and febrile control

\footnotetext{
The first three authors contributed equally to this work.

'Department of Pediatrics, Faculty of Medicine, University of Toyama, Toyama, Japan; ${ }^{2}$ Department of Pharmacology, Graduate School of Science and Engineering for Research University of Toyama, Toyama, Japan; ${ }^{3}$ Department of Pathology, Faculty of Medicine, University of Toyama, Toyama, Japan; ${ }^{*}$ Department of Legal Medicine, Faculty of Medicine, University of Toyama, Toyama, Japan; '5ivision of Cardiology, Department of Pediatrics, University of Utah, Salt Lake City, Utah. Correspondence: Fukiko Ichida (fukiko@med.u-toyama.ac.jp)

Received 29 October 2015; accepted 23 March 2016; advance online publication 25 May 2016. doi:10.1038/pr.2016.93
} 


\section{Articles $\mid$ Ichida et al.}

Table 1. Demographic data of Kawasaki disease (KD) patients and two control groups

\begin{tabular}{|c|c|c|c|c|}
\hline & \multicolumn{2}{|c|}{$\mathrm{KD}(n=23)$} & \multirow[b]{2}{*}{ Febrile $(n=6)$} & \multirow[b]{2}{*}{ Healthy $(n=6)$} \\
\hline & Responder $(n=19)$ & Refractory $(n=4)$ & & \\
\hline Age at onset (m) & $3-47(19)$ & $19-91(40)$ & $8-49(21)$ & $12-40(24)$ \\
\hline CAL (+/-) & $0 / 19$ & $1 / 3$ & $0 / 6$ & $0 / 6$ \\
\hline CRP (mg/dl) & $2.1-22.8(6.3)$ & $9.2-30.2(11.4)$ & $0.1-3.1(1.6)$ & ND \\
\hline Neutrophils (\%) & $36-86(63)$ & $73-92(75)$ & $20-78(51)$ & $31-60(37)$ \\
\hline Platelet count $\left(\times 10^{4} / \mathrm{mm}^{3}\right)$ & $22.5-82.4(34.7)$ & $10.7-39.9(22.7)$ & $24.2-44.0(27.2)$ & $25.3-42.7(33.4)$ \\
\hline $\mathrm{Na}(\mathrm{mmol} / \mathrm{l})$ & $129-140(135)$ & $135-136(136)$ & ND & ND \\
\hline AST (IU/I) & $16-260(40)$ & $24-45(30)$ & $24-63(32)$ & $29-51(44)$ \\
\hline Duration of fever (d) & $3-10(6)$ & $10-15(12)^{*}$ & $2-7(5)$ & ND \\
\hline
\end{tabular}

groups except duration of fever and C-reactive protein levels. There were no significant differences between responders and refractory KD patients except for duration of fever, which was longer in refractory KD patients. All of the KD patients ultimately improved after intravenous immunoglobulin (IVIG) treatment without any adverse effects, albeit after the second IVIG doses or third line therapy using Infliximab in the four refractory KD patients. Only one patient, who had been refractory to the second IVIG treatment, developed CAL during the convalescent stage (Table 1).

\section{Analysis of miR and mRNA Expression}

Part 1: preliminary study. Analysis of the initial four $\mathrm{KD}$ patient samples revealed nine miRs that were significantly different from healthy controls (Table 2). Three (miR-93, miR$150-3 \mathrm{p}$, and miR-877) were down-regulated, while the other six (miR-92b, miR-182, miR-296-5p, miR-320d, miR-494, and miR-1826) were up-regulated. All of them normalized by the convalescent phase (data not shown). Six of the miRs (miR-93, miR-150-3p, miR-877, miR-92b, miR-182, and miR296-5p) were specific to the KD group while the other three were dysregulated in both the KD and febrile groups.

Part 2: Follow-up comparison study between refractory KD patients and responders. We classified three stages for the responder $\mathrm{KD}$ patients, during the febrile stage before the initiation of IVIG treatment (Stage-1), immediately after IVIG infusion during the defervescence stage (Stage-2), and finally during the convalescent phase at $2-4 \mathrm{wk}$ after disease onset (Stage-3). In the refractory patients, all patients were referred to our hospital for second IVIG or third line therapy. Therefore, Stage-1 blood samples were collected before second IVIG or third line therapy during the fever stage, Stage-2 blood samples were collected after these therapies during the defervescence phase, and Stage- 3 blood samples were collected during the convalescent phase at $4 \mathrm{wk}$ after disease onset.
Table 2. Analysis of microRNAs (miRs) in the preliminary study

\begin{tabular}{|c|c|c|c|c|}
\hline Group & $\begin{array}{c}\text { Systematic } \\
\text { name }\end{array}$ & $\begin{array}{l}\text { Fold change } \\
\left(\log _{1.5}\right)\end{array}$ & $P$-value & $\begin{array}{c}\text { Down-/ } \\
\text { upregulation }\end{array}$ \\
\hline KD & hsa-miR-93 & -0.64 & 0.0222 & Down \\
\hline KD & hsa-miR-150-3p & -0.59 & 0.1144 & Down \\
\hline KD & hsa-miR-877 & -0.53 & 0.0638 & Down \\
\hline KD & hsa-miR-92b & 0.66 & 0.0005 & Up \\
\hline KD & hsa-miR-182 & 0.59 & 0.0160 & Up \\
\hline KD & hsa-miR-296-5p & 0.60 & 0.0036 & Up \\
\hline KD and Febrile & hsa-miR-320d & 1.00 & 0.0002 & Up \\
\hline KDand Febrile & hsa-miR-494 & 0.75 & 0.0025 & Up \\
\hline KD and Febrile & hsa-miR-1826 & 0.68 & 0.0129 & Up \\
\hline
\end{tabular}

Analysis of the initial four Kawasaki disease (KD) patient samples revealed the expression of nine of 847 human miRs (1.1\%) that were significantly different from healthy controls. Three (miR-93, miR-150-3p, and miR-877) were down-regulated, while the other six (miR92b, miR-182, miR-296-5p, miR-320d, miR-494, and miR-1826) were up-regulated. All of them normalized by convalescent phase. Six of the miRs (miR-93, miR-150-3p, miR-877, miR-92b, miR-182, and miR-296-5p) were specific to the KD group while the other three (miR-320d, miR-494, and miR-1826) were dysregulated in both the KD and febrile disease groups.

Four of the six miRs (miR-93, miR-150-3p, miR-182, and miR-296), which were differentially expressed in the preliminary study, and an additional two miRs (miR-145-5p and miR$145-3 p)$ previously implicated in KD (17), were selected for further analysis by reverse-transcriptase PCR in the full KD cohort. Each of these miRs have been reported to be involved in immune dysregulation, endothelial damage, and were more highly expressed in the refractory $\mathrm{KD}$ patients than in responders (Figures 1a, 2a,b,3a,c) during at least one stage. Of note, miR150-3p levels were not significantly elevated either in responders or refractory KD patients compared with controls during Stage1 , but were significantly elevated in refractory KD patients during Stage-2, and then normalized by Stage-3 (Figure 3a). These high levels of miRs in refractory KD patients lasted even after alleviation of fever although they peaked at Stage- 2 . 
mRNAs: Functional Category and Pathway Analysis

The hierarchical clustering of differentially expressed gene in PBMCs in KD showed that 669 of the 54,680 genes analyzed $(1.22 \%)$ were significantly up- or down- regulated at the time of diagnosis. Most of these returned to normal after defervescence. The functional category and pathway analysis of the mRNAs identified by the hierarchical clustering of the differentially expressed genes were explored using Ingenuity Pathways Analysis (IPA). This analysis identified networks with known functional relationships and there were several interesting interactions between mRNAs and miRs which were identified to be dysregulated. The expression of SGCZ, EIF4G3, PBX3, ANO4, FAM129B, and LIMCH1 were upregulated and MAPKBP1, AUTS2, DEPDC1 and HIVEP3 were down-regulated: these are associated with coronary artery disease. WASF1, TEC, ITGB1, and CD44 were upregulated, whereas PI3 was down-regulated: these have a role in leukocyte rolling and docking on the endothelial surface of capillaries, or leukocyte transmigration through capillary endothelial layer (Table 3). Analyzing them with IPA indicated VEGF and SGCZ (Sarcoglycan zeta) are directly regulated by miR-93 (Table 4), whereas there are data supporting a role for $\mathrm{VEGF}$ in $\mathrm{KD}$ and coronary artery disease, we are not aware of a role for SGCZ.

Notably, in the KD patients, the changes in expression of VEGFA mRNA were inversely related to the changes in miR93 expression, i.e. mRNA levels were initially elevated but fell Stage-2 while miR levels were initially lower than controls but increased after alleviation of fever (Figures 1a,b,4).

\section{Analysis on Plasma Level of VEGF-A (ELISA)}

To prove that the changes in PBMC mRNA levels of VEGFA influenced the plasma level of VEGF-A protein, plasma levels of VEGF-A were quantitated by enzyme-linked immunosorbent assay (ELISA). As shown in Figure 1c, the plasma levels of VEGF-A were elevated before treatment, but decreased immediately after defevescence in responders, while these levels continued to be high until the recovery stage in the refractory KD group.

\section{DISCUSSION}

In this study, we identified key miRs that modulate gene expression during signaling networks and potential interactions between mRNAs and miRs in patients with acute KD. In addition, gene expression profiling of mRNA was similar to those reported before, which described gene expression profiling in the presence of high-dose IVIG in patients with KD (18).

We identified a small number of miRs that were significantly higher or lower in acute KD patients, and normalized after defervesence. It is interesting that such a small number of known miRs $(<0.7 \%(6 / 847)$ of those investigated) are associated with $\mathrm{KD}$ and that changes in their expression, and the network of mRNAs they control, occur temporally with the response to treatment. In addition, these levels of miRNAs were strikingly high, especially in refractory $\mathrm{KD}$ patients, suggesting these miRNAs are associated with disease severity and may have potential role for the disease progression and responsiveness to the IVIG treatment.

\section{miR-93 and VEGF-A Signaling and Coronary Arteritis}

The expression of miR-93 and VEGFA mRNA in PBMCs showed a strong negative correlation and plasma levels of VEGF-A changed in response to the changes in transcription (Figures 1a-c,4). These findings suggest that miR-93 is inversely related to VEGFA mRNA and plays an important role in pathogenesis in acute $\mathrm{KD}$. The patient with CAL who had the longest duration of fever also had the highest expression of
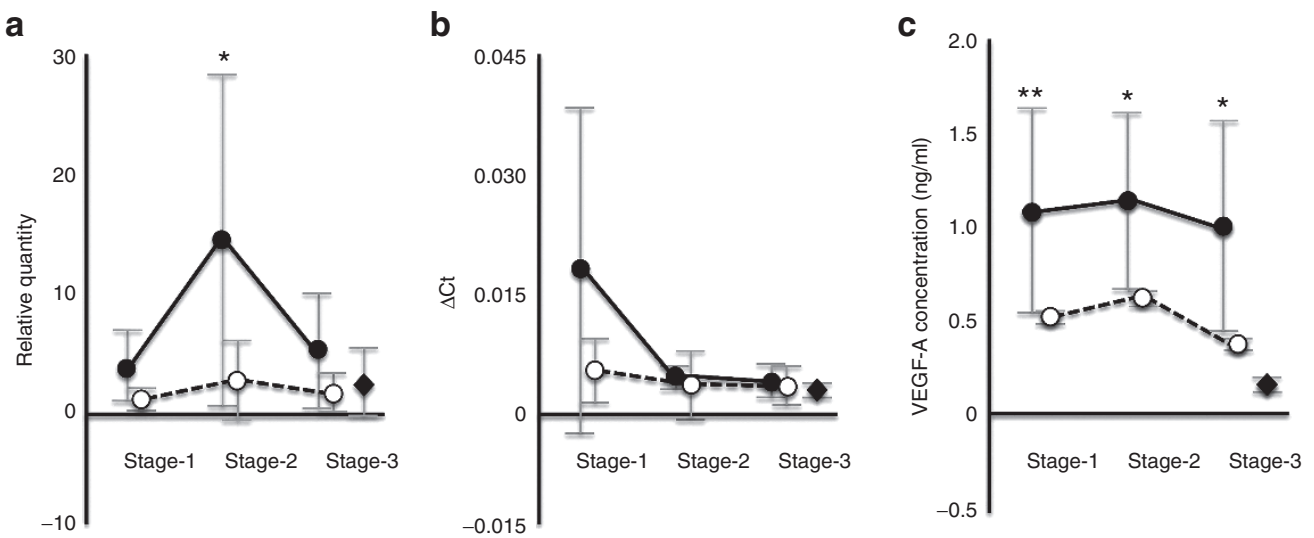

Figure 1. Relationship between miR-93 and VEGFA. The data are presented as mean \pm SD. Dots are mean and whiskers are SD. " $P<0.05$ and ${ }^{* * *} P<0.01$ by $t$-test. Open circle is responder $(n=19)$, closed circle is refractory Kawasaki Disease (KD) $(n=4)$ and closed diamond is control ( $n=12)$. (a) The sequential change of microRNA-93 (miR-93)/RNU48. We normalized the expression of miR-93 to the expression of RNU48. miR-93 expression was higher in the refractory KD patients than in responders and lasted even after alleviation of fever. Especially, miR-93 in the refractory KD patients was significantly higher than that in responders in Stage-2. (b) Change of vascular endothelial growth factor A (VEGFA)/glyceraldehyde 3-phosphate dehydrogenase (GAPDH) mRNA. VEGFA mRNA in refractory KD patients was higher than it in responders. The level of VEGFA mRNA was decreasing at Stage-2 and became normal at Stage-3. The changes in expression of VEGFA mRNA were inversely related to the changes in miR-93 expression, i.e., mRNA levels were initially elevated but fell at Stage 2, while miR levels were initially lower than controls but increased after alleviation of fever. (c) The sequential change of expression of VEGFA. The plasma levels of VEGF-A in the refractory KD patients were significantly higher than that in responders in all Stage. 


\section{Articles | Ichida et al.}

a

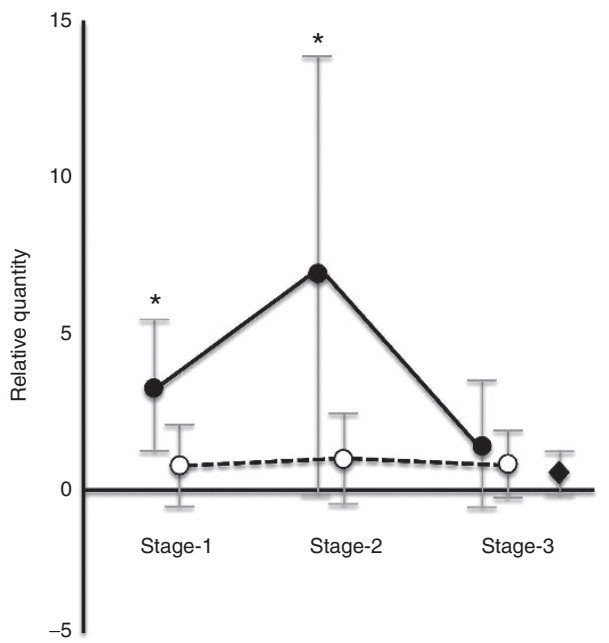

b

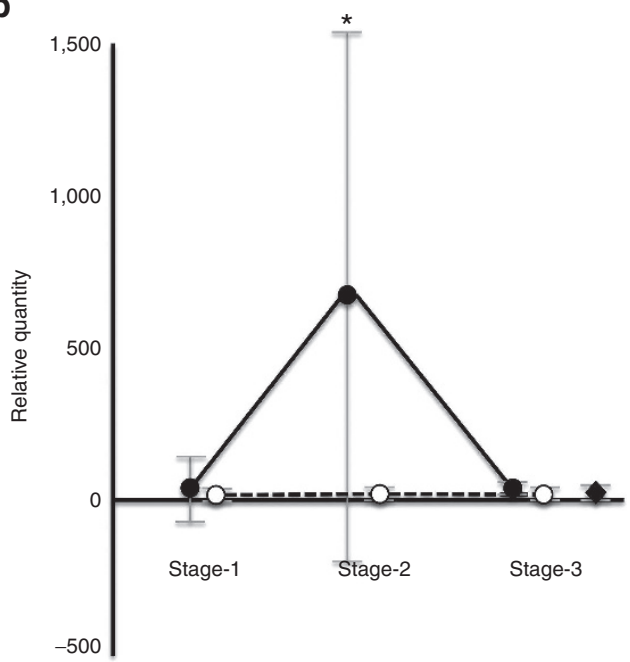

Figure 2. Sequential changes of microRNA (miR)-145-5p and miR-145-3p. The data are presented as mean \pm SD. Dots are mean and whiskers are SD. ${ }^{*} P<0.05$ by $t$-test. Open circle is responder $(n=19)$, closed circle is refractory Kawasaki Disease (KD) $(n=4)$ and closed diamond is control $(n=12)$. (a) The sequential change of miR-145-5p/RNU48. (b) The sequential change of miR-145-3p/RNU48. Two miRs (miR-145-5p, and miR-145-3p) expression was significantly high in refractory KD patients at Stage-2 and becoming normal at Stage-3.

a

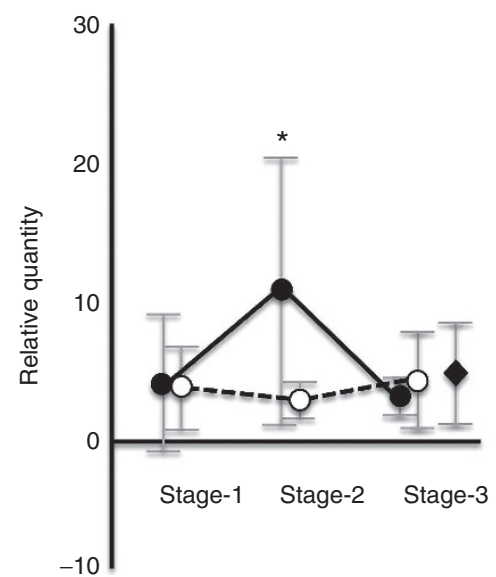

b

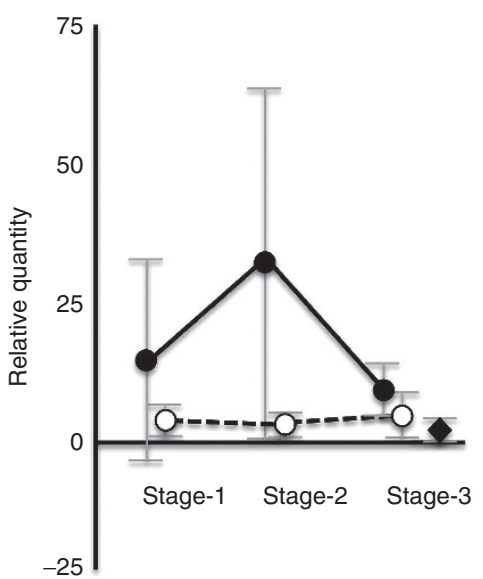

C

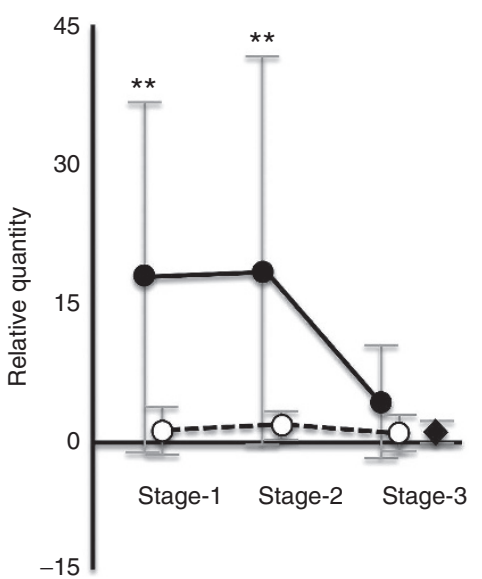

Figure 3. Sequential changes of microRNA (miR) miR-150-3p, miR-182, and miR-296. The data are presented as mean \pm SD. Dots are mean and whiskers are standard deviation. ${ }^{*} P<0.05$ and ${ }^{* *} P<0.01$ by $t$-test. Open circle is responder $(n=19)$, closed circle is refractory Kawasaki Disease (KD) $(n=4)$ and closed diamond is control $(n=12)$. (a) The sequential change of miR-150-3p/RNU48. miR-150-3p in refractory KD patients was significantly higher than that in responders in Stage-2. (b) The sequential change of miR-182/RNU48. miR-182 in refractory KD patients was higher than in responders in all stages. (c) The sequential change of miR-296/RNU48. miR-296 in refractory KD patients was significantly higher than in responders in Stage-1 and Stage-2.

VEGFA mRNA, highest plasma levels of VEGF-A in Stage-1 and Stage-2, and highest levels of miR-93 at Stage-2, suggesting miR-93 and VEGFA mRNA contribute to pathogenesis of coronary artery involvement in acute KD patients. We previously reported that plasma levels of VEGF-A correlate with the development of CAL in KD patients (3). VEGF-A binds to VEGF receptor-1 or VEGF receptor-2 on the surface of vascular endothelium leading to angiogenesis, chemotactic attraction of macrophages, neutrophils, and vasodilation, indirectly by nitric oxygen release $(19,20)$. The human VEGF family has five members, VEGF-A, B, C, D and placenta growth factor, and each of them has tissue-specific roles. The function of VEGF-A has been studied mostly on cells of the vascular endothelium, although it does have various effects on a number of other cell types, such as stimulating monocyte/macrophage migration, neurons, cancer cells, and kidney epithelial cells. In vitro, VEGF-A has been shown to stimulate endothelial cell mitogenesis and cell migration. VEGF-A is also a vasodilator, which increases microvascular permeability and was originally referred to as vascular permeability factor called VEGF before discovering other members $(19,20)$. We have developed a model showing that down-regulation of miR-93 during the acute phase of KD results in increased expression of VEGFA mRNA which inversely related to miR-93.

\section{miR-145-5p, miR-145-3p, and TGF- $\beta$ Signaling Pathway}

In our study, two miRs (miR-145-5p and miR-145-3p) expression was significantly high in refractory KD patients at Stage-2 
Table 3. mRNAs: Functional category

\begin{tabular}{|c|c|c|c|c|}
\hline Gene symbol (protein) & $\begin{array}{c}\text { Kawasaki disease } \\
\text { (Stage-1) }\end{array}$ & Control & $\begin{array}{l}\text { Fold change } \\
\left(\log _{2}\right)\end{array}$ & $\begin{array}{l}\text { Up-/down } \\
\text { regulation }\end{array}$ \\
\hline SGCZ (Sarcoglycan zeta) & 23.4 & 10.1 & 2.33 & Up \\
\hline EIF4G3 (Eukaryotic translation initiation factor 4 gamma 3) & 115.9 & 51.3 & 2.26 & Up \\
\hline PBX3 (Pre-B-cell leukemia transcription factor 3 ) & 17.7 & 7.5 & 2.35 & Up \\
\hline ANO4 (Anoctamin 4) & 18.9 & 9.0 & 2.09 & Up \\
\hline MAPKBP1 (mitogen-activated protein kinase binding protein 1) & 32.3 & 40.0 & 0.80 & Down \\
\hline AUTS2 (autism susceptibility candidate 2 ) & 31.7 & 38.2 & 0.83 & Down \\
\hline DEPDC1 (DEP domain containing 1) & 64.4 & 181.8 & 0.35 & Down \\
\hline HIVEP3 (Human immunodeficiency virus type I enhancer binding protein 3 ) & 26.7 & 49.4 & 0.54 & Down \\
\hline CD44 & 51.7 & 18.9 & 2.74 & Up \\
\hline PI3 (peptidase inhibitor 3 ) & 87.4 & 272.0 & 0.32 & Down \\
\hline
\end{tabular}

The expression of SGCZ, EIF4G3, PBX3, ANO4, FAM129B, and LIMCH1 were up-regulated and MAPKBP1, AUTS2, DEPDC1 and HIVEP3 were down-regulated: these are associated with coronary artery disease. WASP, TEC, PKC, ITGB1 and CD44 were up-regulated, and PI3 was down-regulated: these have a role in leukocyte rolling and docking on the endothelial surface of capillaries, or leukocyte transmigration through capillary endothelial layer.

Table 4. mRNAs related to differentially expressed microRNAs (miRs)

\begin{tabular}{|c|c|c|c|c|c|}
\hline miRs & Gene symbol (protein) & Stage-1 & Stage-2 & Fold change $\left(\log _{2}\right)$ & Regulated mRNA \\
\hline \multirow[t]{4}{*}{ miR-93 } & SGCZ (sarcoglycan zeta) & 23.4 & 4.2 & -2.48 & Down \\
\hline & TSHZ3 (teashirt zinc finger homeobox 3 ) & 604.5 & 406.9 & -0.57 & Down \\
\hline & C10orf140 (chromosome 10 open reading frame 140) & 6.3 & 13.4 & 1.09 & Up \\
\hline & ICK (intestinal cell (MAK-like) kinase) & 3.9 & 12.6 & 1.69 & Up \\
\hline miR-182 & BNC2 (basonuclin 2) & 33.5 & 14.6 & -1.20 & Down \\
\hline
\end{tabular}

Note: Targets of miR-92b, 150-3p, 296-5p, and 877 have not been identified.

Among the many RNAs whose expression was altered in the Kawasaki disease (KD) patients, several have been reported to be regulated by the miRs that were differentially expressed. Examples of these are listed here.

and becoming normal at Stage-3 (Figure 2a,b). Previous studies have implicated the TGF- $\beta$ pathway in disease pathogenesis and generation of myofibroblasts in the arterial wall $(17,21)$. They reported that miR-145-5p, which plays a critical role in the differentiation of neutrophils and vascular smooth muscle cells, was expressed at high levels in blood samples from acute $\mathrm{KD}$ but not adenovirus-infected control patients $(P=0.005)$. Pathway analysis of the predicted targets of the six differentially expressed miRs identified the TGF- $\beta$ pathway as the top pathway regulated by miRNAs in KD. Therefore, they concluded that miR-145-5p may participate, along with other differentially expressed miRs, in regulating expression of genes in the TGF- $\beta$ pathway during the acute illness. In our study, miR-145-5p and miR-145-3p expression was especially high in refractory $\mathrm{KD}$ patients, suggesting that these participate in the regulation of genes which may control responsiveness to the treatment and disease severity of vasculitis.

\section{miR-150 and NF-KB Signaling}

In our study, miR-150-3p levels both in responders and refractory $\mathrm{KD}$ were not significantly elevated as compared with controls during Stage-1. However, miR-150-3p levels in refractory KD elevated significantly during Stage- 2 and normalized by Stage- 3 (Figure 3a). MiR-150 regulates NF- $\mathrm{kB}-$ related inflammation (15). Arteritis in KD is known to involve NF- $\mathrm{kB}$ signaling $(9,16)$. MiR-150 is reported to control inflammation through NF- $\mathrm{KB}$ signaling by acting on IRAK3, MAPK, SGCZ, C10ORF14, and ACCN2 resulting in negative feedback signaling $(9,16)$.

We have previously studied that coronary arteritis in $\mathrm{KD}$ patients and the roles of proteins such as VEGF-A, the S100 protein family, and inducible nitric oxide synthase (3,17-24). Myeloid-related protein8 (also known as S100 calcium-binding protein A8), Myeloid-related protein14 (S100 calcium-binding protein A9), and S100 calcium-binding protein A12 are DamageAssociated Molecular Pattern molecules, which are associated 


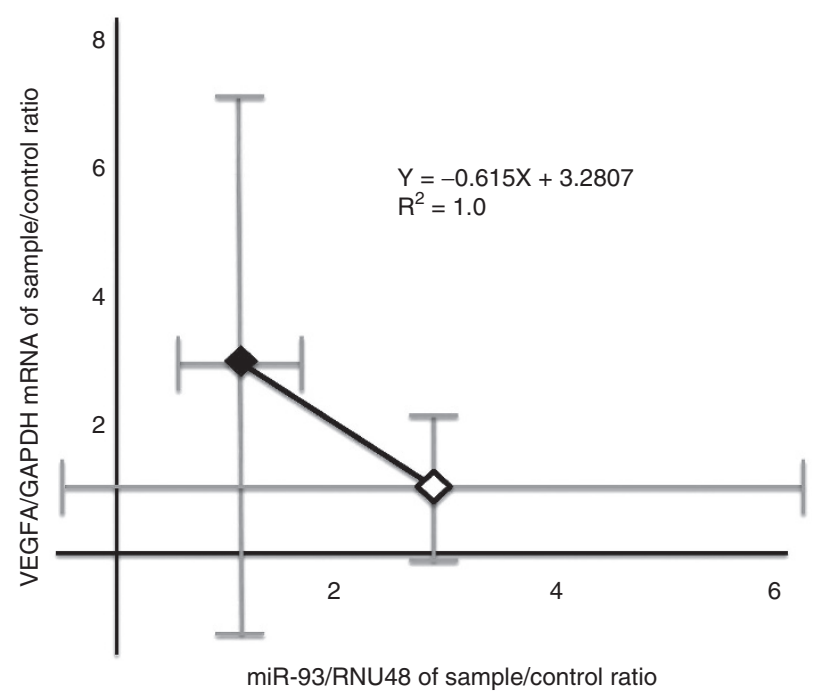

Figure 4. Correlation between normalized vascular endothelial growth factor A (VEGFA) mRNA expression and normalized microRNA-93 (miR-93) expression. We normalized the expression of miR-93 to the expression of RNU48 and then calculated the ratio of expression between patients and controls at each time point. Similarly we normalized the expression of VEGFA to the expression of GAPDH mRNA and then calculated the ratio of expression between patients and controls at each time point. There was a significantly negative correlation between mRNA VEGFA and miR-93. Closed square is Stage- 1 and open diamond is Stage- 2 .

with intracellular signaling pathways by acting as endogenous ligands in the innate immune responses (23-29). Myeloid-related protein8/ Myeloid-related protein14 hetero-dimmers are released in high concentrations at local sites of inflammation and have been described as one of the endogenous ligands of Toll-like receptor 4 . Binding of ligand to Toll-like receptor 4 leads to activation of NF- $\mathrm{kB}$ and induces expression of many proinflammatory cytokines. The serum concentration of receptor for advanced glycation end products may be associated with reactivity to IVIG $(23,28,29)$. Our study showed that miR-150 may play a significant role regulating NF-кB-related inflammation in acute $\mathrm{KD}$.

\section{miR-182 and Immune Dysfunction}

In our study, miR-182 levels were high in refractory $\mathrm{KD}$ patients, and subsequently decreased during the convalescent phase (Figure 3b). MiR-182 was shown to be a key effector of the suppressor activity of regulatory T-cells during an immune response (30). It has been suggested that dysfunctional regulatory T-cells are associated with immune dysfunction in $\mathrm{KD}$, but the mechanisms resulting in T-cell dysfunction remain unknown. Several studies have reported that the numbers of regulatory T-cells decrease and their functional properties are compromised during the acute phase of $\mathrm{KD}$, and partially recover in response to IVIG treatment (31).

\section{miR-296 and Angiogenic Signaling Pathways}

In our study, MiR-296 in refractory KD patients was significantly higher than in responders in Stage- 1 and Stage-2 (Figure 3c). MiRNAs, such as miR-132, miR-126, miR-296,
miR-145, and miR-92a, have been shown to play pro and antiangiogenic roles in the vasculature of both endothelial cells and perivascular cells, acting as key regulators of angiogenesis. However, in pathological situations such as cancer or inflammation, the same angiogenic signaling pathways and miRs are dysregulated and exploited, typically resulting in poorly organized vessels with leaky and tortuous properties (32). miR-296 directly decreased the levels of hepatocytegrowth factor regulated tyrosine kinase substrate, indirectly affected the levels of VEGFR2 and PDGFR $\beta$ by altering their trafficking to the cell surface. Thus, miR-296 may have indirectly affected the levels of VEGFR, resulting in vascular leak of small vessels which is an essential pathological component of vasculitis in acute $\mathrm{KD}$.

\section{Limitations and Suggestion for Further Work}

The major limitation of this study is the small patient cohort. Therefore, it will be important to study larger cohorts, as well as $\mathrm{KD}$ patients who develop CAL, to confirm the role of these miRs and mRNAs in KD progression and pathogenesis. However, we believe these data indicate a significant role for miRs in controlling gene expression and suggest they represent a novel area of investigation.

In this study, we identified key miRs that may modulate gene expression during essential signaling networks in acute $\mathrm{KD}$, however we could not confirm target genes for each of miRs except for VEGF, and we have not established clear causal and mechanistic relationships between the changes in $\mathrm{miR}$ and gene expression. If the predicted target genes for each miRs are confirmed, potential interactions between mRNAs and miRs would be clarified in arteritis of $\mathrm{KD}$.

\section{Conclusions}

In conclusion, we have identified changes in miR profiles in human circulating PBMCs in patients with acute KD. Several miRs appeared to be highly specific to the acute phase of $\mathrm{KD}$, and may participate in regulating expression of genes in the known pathways, such as TGF- $\beta$, VEGF, and NF- $\mathrm{KB}$ signaling. In particular miR-93 appears to control the expression of VEGF-A, and may have an important role in signaling, that results in arteritis in KD.

\section{METHODS}

\section{Subjects and Treatment}

The study population comprised a total of $23 \mathrm{KD}$ patients, six children with unrelated febrile disease (four with viral infections and two with bacterial infections), and six healthy, age-matched children from April 2009 to September 2012 in Toyama University Hospital and related facilities (Table 1-1). All of them were Japanese. Patients were diagnosed with KD if they had five or more of the following six diagnostic criteria $(1,33)$ :

1. High-grade fever that lasted for more than $5 \mathrm{~d}$ if left untreated

2. Red eyes (conjunctivitis) without pus or drainage, also known as "conjunctival injection"

3. Bright red, chapped, or cracked lips, red mucous membranes in the mouth, or strawberry tongue

4. Rash, which may take many forms, but not vesicular (blister-like) 
5. Changes in the extremities: Acute phase-Red palms of the hands and soles of the feet; Later phase-Peeling (desquamation) of palms and soles; peeling may begin around the nails, increased peeling on palms of the hand

6. Swollen lymph nodes (frequently only one lymph node is swollen), particularly in the neck area

Upon diagnosis patients were initially treated with IVIG: $2 \mathrm{~g} / \mathrm{kg} / 24 \mathrm{~h}$ and oral aspirin (ASA: $30 \mathrm{mg} / \mathrm{kg} / \mathrm{d}$ ) with the dose of ASA being decreased to $5 \mathrm{mg} / \mathrm{kg} / \mathrm{d}$, once the fever subsided (34). The patients who responded to IVIG, with recovery from fever within $48 \mathrm{~h}$, are designated responders, while those who did not respond to first or second doses of IVIG are designated refractory patients. These refractory patients were enrolled in the study during this period, as they were referred to our hospital for the second IVIG or third-line treatment. Venous blood samples $(4 \mathrm{ml})$ were obtained three times (Stage-1, Stage-2, and Stage3 ) from the KD patients. Whole blood was collected into sodium EDTA vacuum tubes. Echocardiography and electrocardiograms (ECGs) were performed in the KD patients each time blood was collected to determine whether the patients had coronary artery lesions (CAL, coronary artery diameter Z-score > 2.5). The study was approved by the Ethics Committee of the University of Toyama, performed in accordance with the Declaration of Helsinki, and informed consents were obtained from the parents of the participants.

\section{Isolation of Total RNA from PBMCs}

PBMCs were isolated from whole blood by centrifugation through Histopaque-1077 (Sigma, St. Louis, MO) according to the manufacturer's instructions. Total RNA was extracted from the PBMCs using RNeasy mini kits (Qiagen, K.K., Tokyo, Japan), according to the manufacturer's instructions. Each RNA sample was quality-controlled using a NanoDrop 1,000 (Thermo Fisher Scientific, Wilmington, DE) and the samples were stored at $-150{ }^{\circ} \mathrm{C}$ until further processing.

\section{Analysis of miR and mRNA Expression (Part1: Preliminary Study} for Four Patients with KD)

Gene and miR expression were analyzed using GeneChip systems with the Human Genome U133 Plus 2.0 arrays (Affymetrix, Santa Clara, CA), which were spotted with 54,680 probe sets, and GeneChip miRNA Arrays, which were spotted with 846 probe sets, respectively. A total of 48 arrays were performed: 24 for the KD patients (two arrays per time point, three time points (Stage-1, Stage-2, and Stage3) for each of four patients), 12 for the febrile patients and 12 for healthy controls. Sample preparation for array hybridization was carried out according to the manufacturer's instructions. In short, more than $1 \mu \mathrm{g}$ of total RNA was used to synthesize double-strand cDNA with a 3'IVT Express kit. Biotin-labeled cRNA was then synthesized from the cDNA using FT10AFYB Flash Taq Biotin RNA Labeling Kit (Affymetrix, Santa Clara, CA). After fragmentation, the biotinylated cRNA was hybridized to arrays (35). The arrays were washed, stained with streptavidin-phycoerythrin and scanned with a probe array scanner. The scanned chip was analyzed using GeneChip Analysis Suite software (AGCC 4.3). Hybridization intensity data were converted into a presence/absence call for each gene, and changes in gene expression between experiments were detected by comparison analysis. The data were further analyzed using GeneSpring GX 11.0 software (Silicon Genetics, Redwood City, CA) (35).

\section{Microarray Data Analysis and Statistical Analysis}

In order to identify mRNAs and miRs with expression levels that were different in $\mathrm{KD}$ patients, by comparison with the febrile group and healthy controls, normalized signal intensities were subjected to two-sample $t$-test statistics (applying a nominal significance level of $P<0.01)$. Quantitative $\log _{2}$ metrics were used for the mRNA data and $\log _{1.5}$ for the miR data to quantify expression changes. Probes for nonhuman miRs were omitted.

The data were analyzed using IPA tools (Ingenuity Systems, Mountain View, CA), a web-based application that enables the discovery, visualization and exploration of molecular interaction networks in gene expression data. The gene lists identified by GeneSpring, containing Affymetrix gene ID and natural legalism, were uploaded into IPA. Several algorithms, namely TargetScan 4.1, PicTar, miRanda, and DIANA microT were used for analyzing miR targets. In addition, miRBase, miRGen, and TarBase were used if needed (36-38).

\section{The Replication Study of miR and mRNA Expression (Part2: Sequential Comparison Study Between Responders and Refractory KD Patients)}

Four miRs (miR-93, miR-150-3p, miR-182, and miR-296), which were determined to be differentially expressed in the preliminary study and two miRs (miR-145-5p and miR-145-3p) previously implicated in KD (17), were selected for further analysis. KD patients were divided into two groups: responders to IVIG, $n=19$ and refractory KD patients, $n=4$. The sequential changes of $\mathrm{miR}$ and $\mathrm{mRNA}$ expression were evaluated at three time points (Stage-1, Stage-2, and Stage-3) for each patient and compared with RNA isolated from six healthy controls. In this case, miR expression was analyzed using TaqMan miR Assays while VEGFA mRNA was analyzed by reverse-transcriptase PCR: forward primer (GAGCCTTGCCTTGCTGCTCTAC) and reverse primer: (CACCAGGGTCTCGATTGGATG).

\section{Analysis on Plasma Level of VEGF-A by ELISA}

Plasma concentrations of VEGF-A were determined by a sandwich ELISA using commercial kits (Immuno-Biologic Laboratories, Fujioka, Japan), according to the manufacturer's instructions, in all $\mathrm{KD}$ patients and controls.

\section{Statistics}

Statistical analyses were performed using $t$-test and ANOVA with GraphPad Prism 6.04 (GraphPad Software, La Jolla, CA). The results were considered statistically significant at $P<0.05$. The data are presented as mean $\pm \mathrm{SD}$.

\section{ACKNOWLEDGMENTS}

We thank our colleagues and collaborating hospitals for always supporting us: Hitoshi Moriuchi, Chikako Sakai, Ikuo Hashimoto, Kentaro Tamura, Kiyoshi Hatasaki, Kentaro Shinozaki, Yasunori Ito, Hisano Nakatsubo, Satoshi Umekawa, Syunji Ishihara, Tatsuya Fuchizawa, Junko Yamamoto, Keiichiro Uese, Osamu Higuchi, Motokazu Nakabayashi, Takashi Kuramoto, Shinichi Tsubata, Seiko Saito, Taketoshi Yoshida, Hirokazu Kanegane, Keisuke Ohtsubo, Toshiko Itazawa, and Yuichi Adachi.

\section{STATEMENT OF FINANCIAL SUPPORT}

This study was supported by grants from the Ministry of Education, Culture, Sports, Science and Technology in Japan (Grant-in-Aid for Scientific Research No. 22790969, 2010-2011), RAISE (randomized controlled trial assess immunoglobulin plus steroid efficacy for Kawasaki disease) study grants in Japan 2009-2011, the 15 ${ }^{\text {th }}$ Japan Therapeutic Study Group for Kawasaki Disease in Japan (2010), Takeda Science Foundation in Japan (2009) and Japanese Kawasaki Disease Research Center (2014).

Disclosure: The authors have nothing to disclose.

\section{REFERENCES}

1. Kawasaki T, Kosaki F, Okawa S, Shigematsu I, Yanagawa H. A new infantile acute febrile mucocutaneous lymph node syndrome (MLNS) prevailing in Japan. Pediatr 1974;54:271-6.

2. Terai M, Yasukawa K, Narumoto S, Tateno S, Oana S, Kohno Y. Vascular endothelial growth factor in acute Kawasaki disease. Am J Cardiol 1999;83:337-9.

3. Hamamichi Y, Ichida F, Yu X, et al. Neutrophils and mononuclear cells express vascular endothelial growth factor in acute Kawasaki disease: its possible role in progression of coronary artery lesions. Pediatr Res 2001;49:74-80.

4. Greco A, De Virgilio A, Rizzo MI, et al. Kawasaki disease: an evolving paradigm. Autoimmun Rev 2015;14:703-9.

5. Wang Y, Wang W, Gong F, et al. Evaluation of intravenous immunoglobulin resistance and coronary artery lesions in relation to Th1/Th2 cytokine profiles in patients with Kawasaki disease. Arthritis Rheum 2013;65: 805-14.

6. Jia S, Li C, Wang G, Yang J, Zu Y. The T helper type 17/regulatory T cell imbalance in patients with acute Kawasaki disease. Clin Exp Immunol 2010;162:131-7. 


\section{Articles $\mid$ Ichida et al.}

7. Crome SQ, Wang AY, Levings MK. Translational mini-review series on Th17 cells: function and regulation of human T helper 17 cells in health and disease. Clin Exp Immunol 2010;159:109-19.

8. Afzali B, Mitchell P, Lechler RI, John S, Lombardi G. Translational minireview series on Th17 cells: induction of interleukin-17 production by regulatory T cells. Clin Exp Immunol 2010;159:120-30.

9. Ichiyama T, Yoshitomi T, Nishikawa M, et al. NF-kappaB activation in peripheral blood monocytes/macrophages and T cells during acute Kawasaki disease. Clin Immunol 2001;99:373-7.

10. Cursiefen C, Chen L, Borges LP, et al. VEGF-A stimulates lymphangiogenesis and hemangiogenesis in inflammatory neovascularization via macrophage recruitment. J Clin Invest 2004;113:1040-50.

11. Kroll J, Waltenberger J. VEGF-A induces expression of eNOS and iNOS in endothelial cells via VEGF receptor-2 (KDR). Biochem Biophys Res Commun 1998;252:743-6.

12. Kaneda R, Fukuda K. MicroRNA is a new diagnostic and therapeutic target for cardiovascular disease and regenerative medicine. Circ J 2009;73: $1397-8$.

13. Kawashima T, Shioi T. MicroRNA, emerging role as a biomarker of heart failure. Circ J 2011;75:268-9.

14. De Rosa S, Curcio A, Indolfi C. Emerging role of microRNAs in cardiovascular diseases. Circ J 2014;78:567-75.

15. Sonkoly E, Ståhle M, Pivarcsi A. MicroRNAs and immunity: novel players in the regulation of normal immune function and inflammation. Semin Cancer Biol 2008;18:131-40.

16. Ichiyama T, Ueno $Y$, Hasegawa M, Niimi A, Matsubara T, Furukawa S. Intravenous immunoglobulin inhibits NF-kappaB activation and affects Fcgamma receptor expression in monocytes/macrophages. Naunyn Schmiedebergs Arch Pharmacol 2004;369:428-33.

17. Shimizu C, Kim J, Stepanowsky P, et al. Differential expression of miR-145 in children with Kawasaki disease. PLoS One 2013;8:e58159.

18. Abe J, Jibiki T, Noma S, Nakajima T, Saito H, Terai M. Gene expression profiling of the effect of high-dose intravenous Ig in patients with Kawasaki disease. J Immunol 2005;174:5837-45.

19. Stacker SA, Vitali A, Caesar C, et al. A mutant form of vascular endothelial growth factor (VEGF) that lacks VEGF receptor-2 activation retains the ability to induce vascular permeability. J Biol Chem 1999;274:34884-92.

20. Larrivée B, Karsan A. Signaling pathways induced by vascular endothelial growth factor (review). Int J Mol Med 2000;5:447-56.

21. Shimizu C, Jain S, Davila S, et al. Transforming growth factor-beta signaling pathway in patients with Kawasaki disease. Circ Cardiovasc Genet 2011;4:16-25.

22. Yu X, Hirono KI, Ichida F, et al. Enhanced iNOS expression in leukocytes and circulating endothelial cells is associated with the progression of coronary artery lesions in acute Kawasaki disease. Pediatr Res 2004;55: $688-94$.
23. Foell D, Ichida F, Vogl T, et al. S100A12 (EN-RAGE) in monitoring Kawasaki disease. Lancet 2003;361:1270-2.

24. Hirono K, Foell D, Xing Y, et al. Expression of myeloid-related protein-8 and -14 in patients with acute Kawasaki disease. J Am Coll Cardiol 2006;48:1257-64.

25. Burns JC. S100 proteins in the pathogenesis of Kawasaki disease. J Am Coll Cardiol 2006;48:1265-7.

26. Wittkowski H, Hirono K, Ichida F, et al. Acute Kawasaki disease is associated with reverse regulation of soluble receptor for advance glycation end products and its proinflammatory ligand S100A12. Arthritis Rheum 2007;56:4174-81.

27. Foell D, Wittkowski H, Vogl T, Roth J. S100 proteins expressed in phagocytes: a novel group of damage-associated molecular pattern molecules. J Leukoc Biol 2007;81:28-37.

28. Ye F, Foell D, Hirono KI, et al. Neutrophil-derived S100A12 is profoundly upregulated in the early stage of acute Kawasaki disease. Am J Cardiol 2004;94:840-4.

29. Viemann D, Strey A, Janning A, et al. Myeloid-related proteins 8 and 14 induce a specific inflammatory response in human microvascular endothelial cells. Blood 2005;105:2955-62.

30. Kelada S, Sethupathy P, Okoye IS, et al. miR-182 and miR-10a are key regulators of Treg specialisation and stability during Schistosome and Leishmania-associated inflammation. PLoS Pathog 2013;9:e1003451.

31. Ni FF, Li CR, Li Q, Xia Y, Wang GB, Yang J. Regulatory T cell microRNA expression changes in children with acute Kawasaki disease. Clin Exp Immunol 2014;178:384-93.

32. Anand S, Cheresh DA. Emerging role of Micro-RNAs in the regulation of angiogenesis. Genes Cancer 2011;2:1134-8.

33. Kawasaki T. Clinical signs and symptoms of mucocutaneous lymph node syndrome (Kawasaki disease). Jpn J Med Sci Biol 1979;32:237-8.

34. JCS Joint Working Group. Guidelines for diagnosis and management of cardiovascular sequelae in Kawasaki disease (JCS 2013). Digest version. Circ J 2014;78:2521-62.

35. Takasaki I, Takarada S, Fukuchi M, Yasuda M, Tsuda M, Tabuchi Y. Identification of genetic networks involved in the cell growth arrest and differentiation of a rat astrocyte cell line RCG-12. J Cell Biochem 2007;102:1472-85

36. Kiriakidou M, Nelson PT, Kouranov A, et al. A combined computationalexperimental approach predicts human microRNA targets. Genes Dev 2004;18:1165-78.

37. Megraw M, Sethupathy P, Corda B, Hatzigeorgiou AG. miRGen: a database for the study of animal microRNA genomic organization and function. Nucleic Acids Res 2007;35(Database issue):D149-55.

38. Sethupathy P, Corda B, Hatzigeorgiou AG. TarBase: A comprehensive database of experimentally supported animal microRNA targets. RNA 2006;12:192-7. 